\title{
EMPLEABILIDAD DE LOS GRADUADOS DE LA CARRERA DE INGENIERÍA DE SISTEMAS DE LA UNIVERSIDAD POLITÉCNICA SALESIANA DE GUAYAQUIL, DEL AÑO 2017 AL 2019
}

EMPLOYABILITY OF THE GRADUATES OF THE SYSTEMS ENGINEERING CAREER OF THE SALESIANA POLITECHNICAL UNIVERSITY OF GUAYAQUIL, FROM THE YEAR 2017 TO 2019

\section{Bertha Alice Naranjo Sánchez ${ }^{1}$}

Sindy Tatiana Gómez Pihuave ${ }^{2}$

Salesiana Politechnical University Of Guayaquil

\section{RESUMEN}

El presente artículo tiene como objetivo analizar la empleabilidad de los graduados de la carrera Ingeniería de Sistemas de la Universidad Politécnica Salesiana sede Guayaquil, durante tres periodos comprendidos entre el año $2017 \mathrm{y}$ 2019. La investigación es de tipo descriptiva con un enfoque cuali-cuantitativo de corte longitudinal

$1 \quad$ Alice Naranjo. Universidad Politécnica Salesiana, Sede Guayaquil. Chambers \#227 y 5 de Junio, Guayaquil Telf. 042590630 Ext. 4550

s/n CELULAR. berthanaranjo.ups.edu.ec https://orcid.org/0000-0002-4386-2335

2 Sindy Gómez P. berthanaranjo.ups.edu.ec Universidad Politécnica Salesiana, Sede Guayaquil 0000-0003-4831-866X

en relación a los años de revisión, la población de estudio corresponde a 188 graduados, de los cuales se analizó una muestra probabilística de 127 profesionales que respondieron una encuesta online creada para el efecto, se realizó además la prueba de independencia y homogeneidad a través del método del chicuadrado para variables objeto de análisis. Los resultados obtenidos en empleabilidad son alentadores para los graduados de la Carrera de ingeniería en Sistemas independientemente del género con una alta satisfacción en la inserción laboral. 


\section{PALABRAS CLAVE}

Empleabilidad, Ingeniería en Sistemas, equidad de género, satisfacción laboral.

\section{ABSTRACT}

This article aims to analyze the employability of graduates of the Systems Engineering career of the Salesian Polytechnic University in Guayaquil, during three periods between 2017 and 2019. The research is descriptive in nature with a qualitative-quantitative approach of longitudinal cutting in relation to the years of revision, the study population corresponds to 188 graduates, of whom a probabilistic sample of 127 professionals who responded to an online survey created for the effect, also carried out the test of independence and homogeneity through the chi-square method for variables object of analysis. The results obtained in employability are encouraging for graduates of the Engineering Career in Systems regardless of gender with

\section{KEYWORDS}

Employability, Systems Engineering, Gender Equity, Job Satisfaction.

\section{INTRODUCCIÓN}

Actualmente en el Ecuador existen mayores oportunidades para el acceso a la educación superior. Según la Senescyt, en un informe del primer semestre del 2020, las instituciones de educación superior ofertaron 113.072 cupos, es decir un $31 \%$ más que en el primer semestre del 2019, las provincias que registraron mayor incremento de cupos fueron: Manabí, Guayas, Los Ríos y Chimborazo, las instituciones de educación superior correspondieron al 95\% de instituciones públicas y el $5 \%$ de instituciones privadas, 218 carreras vigentes fueron ofertadas en distintas jornadas y modalidades, de las cuales 144 fueron de Universidades y Escuelas Politécnicas públicas y 74 pertenecían

a institutos superiores técnicos y tecnológicos (Senecyt, 2020).

Con esta amplia oferta, las Universidades e institutos tecnológicos brindan a los jóvenes universitarios la posibilidad de acceder a un empleo seguro desarrollando competencias que le habilitarían para ejercer con un título profesional labores dentro de las empresas públicas o privadas del sector dentro del departamento relacionado a su ejercicio profesional. Teniendo en cuenta que la empleabilidad es la aptitud para realizar una tarea determinada del sector productivo dentro del mercado de trabajo, ésta se centra en las oportunidades de empleo adecuado y la capacidad de mejorar la situación laboral de los jóvenes que se preparan en las Universidades.

La realidad nacional acerca de la empleabilidad en nuestro medio, es un factor que debe de ser observado minuciosamente por las Instituciones de Educación Superior y se ha convertido en una de las principales responsabilidades de estas Instituciones, debido a que es indispensable que se empleen acciones con el propósito de indagar las posibilidades de empleo adecuados para los profesionales graduados.

La Universidad Politécnica Salesiana, Sede Guayaquil, con un alto sentido de responsabilidad social cuenta con la Unidad de Vinculación con la Sociedad, que entre otras funciones realiza el seguimiento a graduados respecto a su situación laboral, y a través de la Bolsa de Trabajo, facilita la inserción laboral de estos exponiendo sus servicios a diferentes organizaciones laborales.

La presente investigación se centra en el seguimiento de los graduados de la carrera de Ingeniería de Sistemas del periodo comprendido entre el 2017 y el 2019, tiene como objetivo principal analizar la empleabilidad, considerando distintos factores internos y externos que permitan conocer la situación laboral de los graduados de la Universidad. 


\section{MARCO TEÓRICO}

\section{Ingeniería de Sistemas}

La ingeniería se ha convertido en un terreno triunfante, debido a que es la encargada de encontrar soluciones prácticas a la vida teniendo en cuenta que es la forma de como la ciencia actúa en el mundo. Sin embargo, hoy en día, la ingeniería ya no existe como tal, como un concepto o terreno general. En los últimos años se ha producido una transformación o una revolución al interior de la ingeniería, la cual ya no solamente transforma a la propia ingeniería, sino, tiene también un impacto serio sobre el conocimiento en sentido amplio tanto como sobre la sociedad y la naturaleza (Valencia, 2004).

La Ingeniería de Sistemas es una rama de la Ingeniería que se encarga del diseño, desarrollo, aplicación y mantenimiento de sistemas informáticos, la cual implica el uso de nociones matemáticas que permiten concretar la aplicación tecnológica de las diferentes teorías, el campo de aplicación es bastante amplio, de hecho resulta fundamental en áreas como; la salud, la biologías, la economía, o la comunicación, debido a que la mayoría de las empresas necesitan tecnología informática para llevar a cabo sus proyectos (Gonzáles, 2020).

La Cumbre Mundial sobre la Sociedad de la Información declaró en el año 2003 el deseo y el compromiso de todas las naciones por enfrentar uno de los más importantes desafíos a escala global, la construcción de una sociedad de la información centrada en la persona, integradora y orientada al desarrollo. Los arquitectos de esta nueva sociedad de la información deben ser principalmente los Ingenieros de Sistemas, los cuales tienen la finalidad de promover las Tecnologías de la Información y las Comunicaciones para el desarrollo de las naciones, construir la infraestructura de la información y las comunicaciones como factor habilitador de la Sociedad de la Información, facilitar el acceso a la información y al conocimiento a todos los hombres y mujeres, crear la capacidad humana para que cada persona tenga la posibilidad de adquirir las competencias y conocimientos para comprender la Sociedad de la Información, fomentar la confianza y seguridad en la utilización de las Tecnologías de las Información, entre otros (Pico, 2015).

La carrera de Ingeniería de Sistemas se configura alrededor de un sistema de competencias profesionales, que se estructuran a través de un conjunto de saberes, conocimientos, habilidades, valores y capacidades para lograr un desempeño profesional competente en los egresados, con responsabilidad profesional y social, de esa manera se comprometen a responder a las demandas cualitativas de la sociedad, el mercado laboral y el contexto de desarrollo local regional y nacional (Cristóbal, 2015).

\section{Capacidades y Habilidades de la Ingeniería de Sistemas}

Los conocimientos y capacidades de un Ingeniero de Sistemas se han vuelto necesarios en diversos campos de acción, múltiples disciplinas y sectores, como entretenimiento, financiero, salud, medioambiente, telecomunicaciones y educación. Existen razones adicionales por las cuales podemos afirmar que la ingeniería de sistemas es la carrera del presente y del futuro: mejores salarios y empleabilidad garantizada. Según los datos del Observatorio Laboral de Colombia para la Educación indica que los profesionales recién graduados de carreras de tecnología de la información presentan una tasa de empleabilidad del $88,10 \%$ y un primer salario mensual bastante atractivo frente a otras especialidades (Novoa, 2016). Además, hay que recalcar que un profesional en Ingeniería de Sistemas, adquiere los conocimientos necesarios para desenvolverse en el ámbito laboral, adquiriendo la capacidad de realizar trabajos complejos desde la creación de sitios 
web, diseño hasta el mantenimiento y sobre todo contribuye al desarrollo de proyectos con carácter informático que aporten al despliegue de servicios y producción en una empresa.

Un profesional en Ingeniería de Sistemas es un profesional global, debido a los conocimientos en temas de tecnología y ciencia para la solución de problemas reales, considerado como un ente transversal capaz de apoyar diversos proceso en lo que respecta al área de ingeniería y matemáticas aplicadas, su formación le permite formularpropuestas paralasolución de problemas reales mediante el uso de la tecnología, lideras proyectos de investigación o empresariales para resolver problemas concretos y sobre todo tomar decisiones con bases sólidas, éticas y científicas en busca del mejoramiento humano, social y tecnológico (UniNorte, 2015). Además, el perfil ocupacional le permite desempeñar sus funciones desde cualquier parte del mundo, con equipos interdisciplinarios y para clientes en todas las áreas, debido a que toda organización necesita profesionales que lideren los procesos de transformación digital, planteen soluciones estratégicas acordes a las necesidades del mercado actual y se adapten a los avances tecnológicos, conjuntamente adquieren la capacidad de generar un impacto positivo en la sociedad.

\section{Empleabilidad}

La empleabilidad se define como la capacidad relativa de un individuo para obtener empleo teniendo en consideración la interacción entre sus características personales y mercado laboral (Rentería-Pérez \& Malvezi, 2008; Lantarón, 2014).

Por otro lado, (Lantarón, 2016), señala que es un término estudiado desde diferentes ópticas y múltiples disciplinas, resultando un concepto complejo que ha ido evolucionando, pues además de poseer una connotación de capacidad de inserción laboral, define una relación entre organizaciones, trabajo y aprendizaje. Basándonos en esto, concluimos que la empleabilidad no puede ser abordada desde una perspectiva única pues es construida a partir de múltiples factores sociales.

Las Instituciones de Educación Superior adquieren un papel fundamental y deben ser capaces de responder a las necesidades del mercado laboral. Desde la perspectiva de la empleabilidad basada en el empleo (demanda), las prácticas más habituales de las Universidades son la integración de prácticas en empresas en el plan de estudio. Desde el punto de vista de la empleabilidad basada en las competencias (oferta), las Universidades responden ofreciendo competencias generales, transversales y específicas, pero también pueden desarrollar otro conjunto de habilidades que son necesarias en los estudiantes y que, en general, se suelen incorporar a través de sus propios programas o bien a través de cursos específicos. Estos dos enfoques de empleabilidad, basada en el empleo y basada en las competencias, son complementarios y responden a un objetivo único, la inserción laboral (Greciano, 2017).

Las Universidades latinoamericanas y específicamente las de Ecuador tienen la responsabilidad de garantizar las competencias necesarias a sus profesionales para ingresar al mercado laboral y ser exitosos laboralmente, por lo que necesita articular mayor vinculación con el sector empresarial y cubrir todas las demandas de la sociedad en su ámbito de acción (Gavilanes \& Fernández, 2015).

La empleabilidad puede definirse actualmente como la competencia que posee una persona para diseñar su carrera profesional, acceder al mercado de trabajo y gestionar su propio desarrollo laboral con acierto y satisfacción. Como sucede con todas las competencias, la competencia para la empleabilidad deberá ponerse de manifiesto en un contexto determinado y perfeccionarse durante toda la vida laboral del sujeto, si bien su aprendizaje y 
desarrollo comenzara desde temprana edad. Por tratarse de una competencia la empleabilidad incluye aspectos relativos al saber, al saber hacer y al saber ser-estar. (González, 2011).

Frente a los retos de la globalización y el surgimiento de nuevas economías basadas en el conocimiento, la educación ha adquirido nuevas características ante la necesidad de elevar la calidad en la formación de los individuos aumentando con ello su nivel de empleabilidad inicial. Diversos organismos internacionales entre ellos, la Organización de las Naciones Unidas para la Educación, la Ciencia y la Cultura (UNESCO), la Organización para la Cooperación Económica y el Desarrollo (OECD) y la Organización Internacional del Trabajo (OIT), etc.; han manifestado como prioritaria la formación de ingenieros altamente competitivos con excelentes capacidades, destrezas y aptitudes laborales, facilitando con ello el crecimiento de la industria en los países, por lo cual, la evaluación del nivel de empleabilidad inicial debe ser una tarea diaria para las Instituciones de Educación Superior permitiendo con esto la detección de áreas de oportunidad en la formación de profesionistas asegurando una fácil inserción al medio laboral (Gonzalez, 2013).

\section{METODOLOGÍA}

Se realizó una investigación descriptiva, de diseño no experimental, longitudinal, debido a que el levantamiento de datos fue realizado en los años de estudios comprendidos entre 2017 a 2019.

La investigación tiene un enfoque cualicuantitativo. La población analizada corresponde a 188 graduados de los cuales sólo 127 accedieron a responder el formulario disponible en Google drive, el cual fue enviado a través de correos electrónicos a los graduados quienes de forma voluntaria accedieron a llenarlo convirtiéndose esta en una muestra probabilística.

El instrumento diseñado constó de 8 preguntas en escala de Likert, tomando en consideración las siguientes dimensiones:

1. Graduados por género

2. Distribución de graduados por situación laboral

3. Motivos por los cuales algunos Graduados no laboran

4. Cargo que ocupan en el trabajo

5. Satisfacción del desempeño laboral

6. Aspectos que representan mayor dificultad para encontrar trabajo

7. Tiempo de trabajo en el ámbito laboral

8. Tipo de empresa en la que laboran

Para el procesamiento de datos se utilizó la estadística descriptiva, mediante la aplicación del Software SPSS.

\section{RESULTADOS}

Mediante el análisis de los datos, se identificó que, del total de la muestra de graduados de la carrera de Ingeniería de Sistemas, el $24,4 \%$ corresponden al período 2017 , el $21,3 \%$ pertenecen al período 2018 y finalmente el $54,3 \%$ corresponden al período 2019. Analizando la cantidad de estudiantes durante el período de análisis, se evidencia que existe un incremento de estudiantes en la Carrera en esos años y por consiguiente un incremento en el número de graduados, tal como se aprecia en la figura 1. 


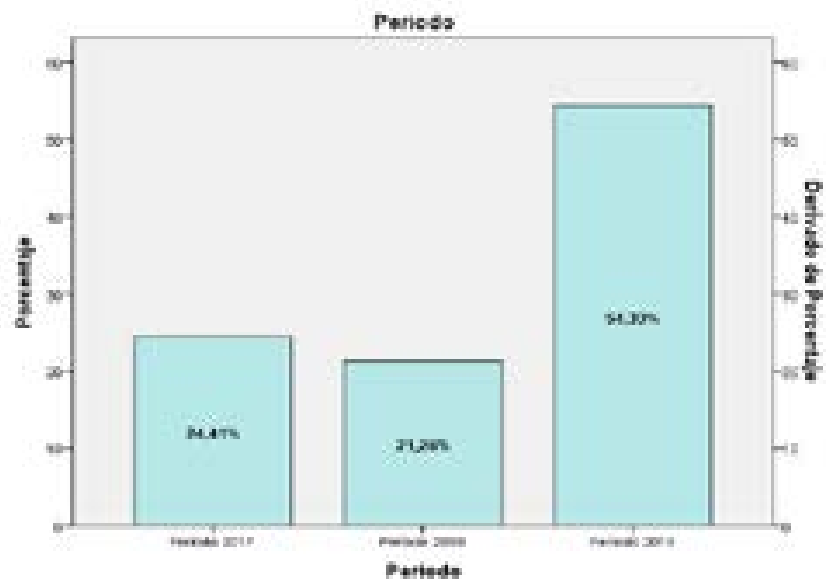

Figura 1 Distribución de graduados según el período

Fuente: Encuestas

\section{Elaborado por: Autores}

En relación al género de los graduados, se identifica que en el período 2017 el que predomina es el masculino con un $58,1 \%$, mientras que el género femenino representa el $41,9 \%$. Durante el período 2018, se ha incrementado el número de estudiantes graduados de género

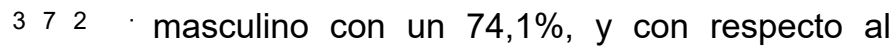
género femenino ha disminuido a un $25,9 \%$. Finalmente, en el período 2019, prevalece el género masculino con un $79,7 \%$, mientras que el género femenino se ha reducido a un 20,3\%. En este contexto se determina que la mayoría de los estudiantes graduados perteneciente a la carrera de Ingeniería de Sistemas dentro del periodo de estudio son de género masculino, por lo que es indispensable que se promocione la oferta académica basada en la inclusión de las mujeres en esta área de estudio (Ver Figura 2).

Figura 2 Graduados por género

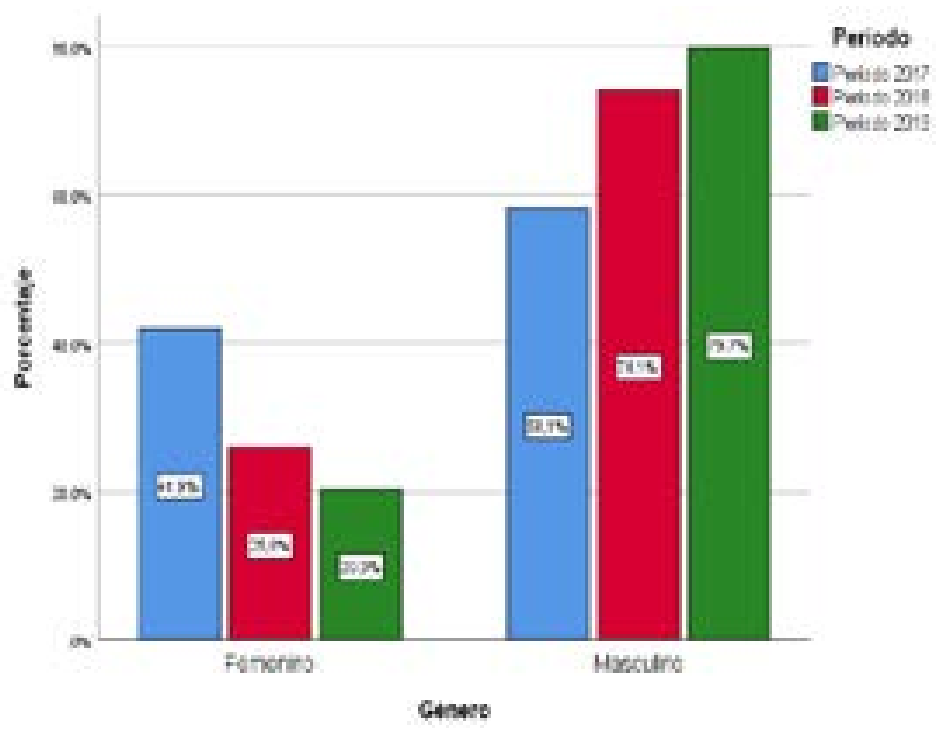

Fuente: Encuestas

Elaborado por: Autores 
Con respecto a la distribución de graduados por situación laboral, en base al análisis realizado, se identificó que en el período 2017 , del $100 \%$ de los estudiantes, el $93,5 \%$ ha ingresado a trabajar, mientras que sólo el $6,5 \%$ aún no poseen trabajo, teniendo en cuenta que en términos absolutos corresponde a 2 estudiantes graduados. En relación al 2018, se determinó que el $74 \%$ accedieron a una fuente de empleo, sin embargo, el 25,9\% aún no obtienen un trabajo, y con respecto al 2019, se observó que, del total de alumnos de este período, el $85,5 \%$ asisten a su lugar de trabajo, mientras que el $14,5 \%$ indicaron que aún no encuentran empleo, teniendo en cuenta que, en términos absolutos de los 69 graduados, diez de ellos aún no encuentran un trabajo estable, tal como se observa en la Figura 3.

Figura 3 Distribución de Graduado por situación laboral

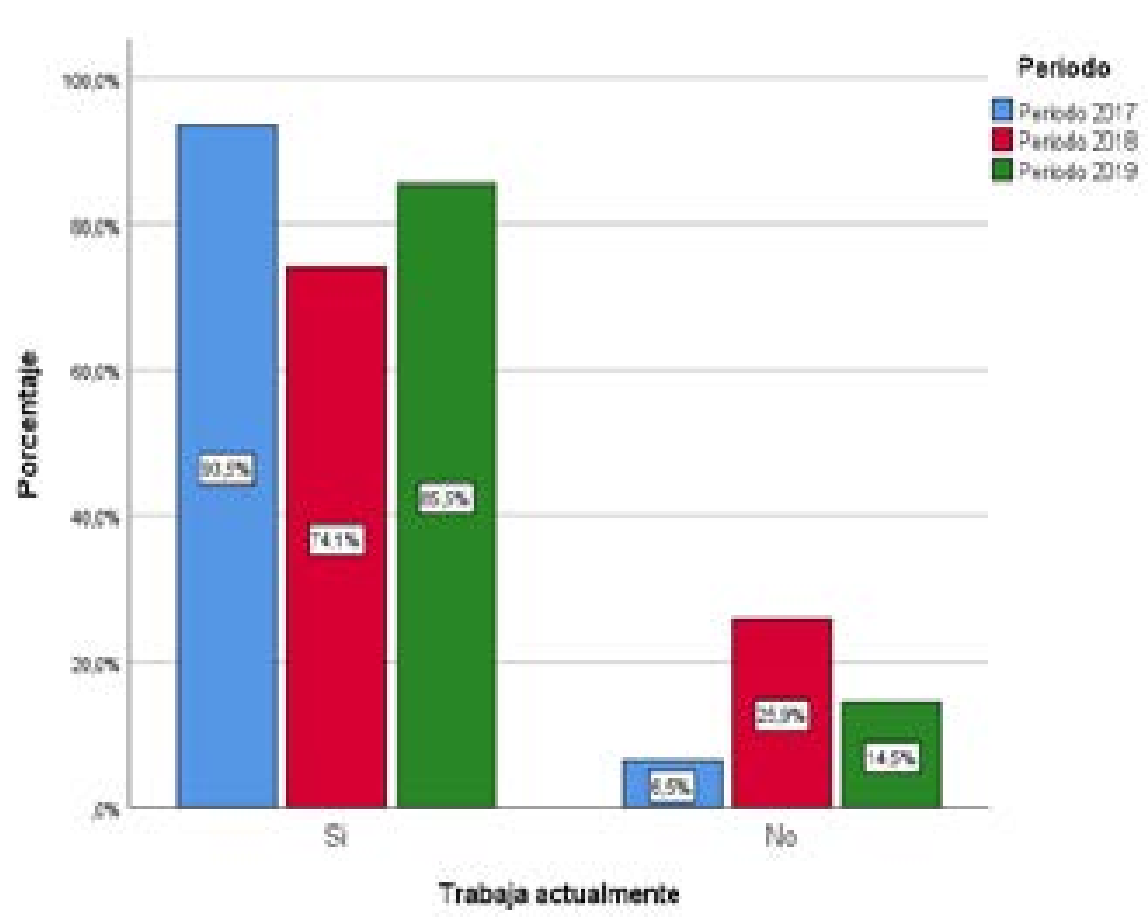

Fuente: Encuestas

Elaborado por: Autores

De los graduados que no se encuentran en situación laboral, se planteó una variable sobre los motivos por los que no encuentran laborando. En este sentido, se identificó que el principal motivo en el período 2017 y 2018 pertenece a motivos personales y que no encuentran empleo, y finalmente en el periodo 2019, la causa principal es problemas de salud y temor al contagio, que pueden ser generados por la pandemia del COVID 19. Este trabajo de relevamiento de información se desarrolló a inicios de Diciembre del 2019, fecha en que la pandemia se hizo presente en el mundo.

En relación a los cargos que desempeñan los graduados en su ámbito laboral, se identificó que del $100 \%$ de los puestos de trabajo que ocupan los graduados en los tres períodos, el $80,6 \%$ corresponde a los cargos operativos seguido por el cargo de jefes departamentales con un $13,9 \%$, mientras que el área gerencial representa el $5,6 \%$, teniendo en cuenta que, 
en términos absolutos de los 108 estudiantes graduados de la carrera de Ingeniería de Sistema que laboran, solo 6 de ellos tienen un cargo ocupacional de alto nivel.

Respecto al grado de satisfacción de los estudiantes en su ámbito laboral, en los tres años se determinó que el $40,7 \%$ del total de los tres años están muy satisfechos con las funciones que desempeñan en su lugar de trabajo, el $25 \%$ están algo satisfechos, el $13,9 \%$ no están ni satisfechos ni insatisfechos, el $13,0 \%$ se encuentran algo insatisfechos y el 7,4\%, están insatisfechos en su lugar de trabajo. Se tomó en cuenta únicamente a los estudiantes que trabajan siendo la población de 108 estudiantes que trabajan mientras que los otros 19 no trabajan y no son tomados en cuenta en el análisis.

De los resultados obtenidos en base a la dificultad que se presenta al momento de buscar empleo, se destaca que en el período 2017 era más difícil conseguir empleo, en el período 2018 era más difícil acceder a un puesto de trabajo, y finalmente en el período 2019, establecieron que era muy difícil encontrar empleo, lo cual denota que en los últimos tres años conseguir empleo ha sido difícil en el país.

En la Tabla 8 relacionada al tiempo de permanencia en su lugar de trabajo, se identificó que en el período 2017 , un $53,8 \%$ tienen de permanencia en su empleo de 9 a 12 meses, en el período 2018 , se identificó con el $30 \%$ a las personas que tienen menos de 3 meses laborando, seguido del $28,6 \%$ que representa a los graduados que están desempeñando sus funciones de 1 a 2 años, y finalmente en el período 2019 , el $70,6 \%$ corresponden a las personas que laboran más de dos años. En este contexto, se determinó que la mayor parte de los graduados que respondieron la encuesta están iniciando su relación laboral.
En relación al tipo de empresa en la que laboran los graduados de la carrera de Ingeniería de Sistemas, se identificó que, en todos los años, los graduados trabajan más en empresas privadas que en empresas públicas y que en el año 2019 se observa graduados laborando en empresas familiares.

\section{PRUEBA DE INDEPENDENCIA Y HOMOGENEIDAD}

Para la prueba de Independencia las hipótesis son:

- $\quad H_{0}$ : No hay relación entre el género de los graduados y el resultado obtenido en relación a su situación laboral actual.

- $\quad \mathrm{H}_{\mathrm{a}}$ : Si hay relación entre el género de los graduados y la situación laboral.

Para la prueba de homogeneidad las hipótesis son:

- $H_{0}$ : la proporción de satisfacción en el ámbito laboral es igual sin tener en cuenta el tipo de empresa en la que trabajan.

- $\mathrm{H}_{\mathrm{a}}$ : La proporción de satisfacción en el ámbito laboral depende del tipo de empresa en la que laboran. 
Tabla 2 Prueba de Independencia

Pruebas de chi-cuadrado

\begin{tabular}{|c|c|c|c|c|c|}
\hline & Valor & df & $\begin{array}{l}\text { Significación } \\
\text { asintótica } \\
\text { (bilateral) }\end{array}$ & $\begin{array}{l}\text { Significación } \\
\text { exacta } \\
\text { (bilateral) }\end{array}$ & $\begin{array}{l}\text { Significación } \\
\text { exacta } \\
\text { (unilateral) }\end{array}$ \\
\hline Chi-cuadrado de Pearson & $1,156^{a}$ & 1 & ,282 & & \\
\hline $\begin{array}{l}\text { Corrección de } \\
\text { continuidad }^{\mathrm{b}}\end{array}$ & ,631 & 1 & ,427 & & \\
\hline Razón de verosimilitud & 1,095 & 1 & ,295 & & \\
\hline Prueba exacta de Fisher & & & & ,277 & ,210 \\
\hline $\begin{array}{l}\text { Asociación lineal por } \\
\text { lineal }\end{array}$ & 1,147 & 1 & ,284 & & \\
\hline N de casos válidos & 127 & & & & \\
\hline
\end{tabular}

Fuente: Encuestas

Elaborado por: Autores

Tabla 3 Prueba de Independencia y Homogeneidad

Pruebas de chi-cuadrado

\begin{tabular}{ll|l|l} 
& & & \\
& Valor & df & Significación asintótica (bilateral) \\
\hline Chi-cuadrado de Pearson & $16,195^{\mathrm{a}}$ & 16 &, 439 \\
\hline Razón de verosimilitud & 21,480 & 16 &, 161 \\
\hline Asociación lineal por lineal &, 041 & 1 &, 839 \\
\hline N de casos válidos & 91 & &
\end{tabular}

a. 20 casillas $(80,0 \%)$ han esperado un recuento menor que 5 . El recuento mínimo esperado es, 13 .

Fuente: Encuestas

Elaborado por: Autores 
En la tabla 2 se muestra que el valor de $P$-Value es 0,282 es decir es mayor que 0,05 , por lo que se identifica que la hipótesis nula de independencia entre las variables es aceptada. Por lo que se deduce que no existe asociación entre el tipo el género y la relación laboral. En relación a la hipótesis nula de homogeneidad en la tabla 3 se muestra el valor obtenido, que corresponde a 0,439 , es decir es mayor a 0,05 , por lo que se acepta la hipótesis nula, y se determina que la proporción de la satisfacción en su ámbito de trabajo es la misma para los graduados de la carrera de Ingeniería de Sistemas, sin tomar en cuenta el tipo de empresa en la que laboran.

Para determinar la tasa de inserción laboral se tomará como base de cálculo la recomendación de "la Organización Internacional del Trabajo (OTI) y la Organización para la Cooperación y el Desarrollo Económico (OCED), que hacen referencia a la relación entre las personas con empleo expresado como porcentaje de la población de edad de trabajar" (INEC, 2016, pág. 14).

Del total de la muestra se estableció que la tasa de inserción laboral es del $22,8 \%$ para el año 2017, 15,7\% para el año 2018 y para el 2019 fue de $46,5 \%$. Esto señala el déficit de la inserción laboral al transcurso de los últimos años, donde ya se estableció la etapa de crisis para el país.

\section{DISCUSIÓN Y ANÁLISIS}

Al obtener los resultados de la encuesta realizada a los graduados de la carrera de Ingeniería de Sistemas se identificó que gran parte de los estudiantes pertenecen el género masculino, se entendería que esta situación de poca participación del género femenino en la Carrera obedece aún a diversos factores que impiden una mayor equidad en participación del género femenino en carreras técnicas (Espinosa, 2018).

De los datos analizados, se obtuvo que de los tres periodos correspondientes a 2017, 2018 y 2019 , el $85 \%$ mantienen una relación laboral, mientras que el $14 \%$ de toda la muestra actualmente no tienen trabajo y que esta situación no está relacionada a competencias que otorga la Carrera, sino que se debe en su mayoría a problemas familiares, problemas de salud $u$ otros conceptos.

En relación a los cargos que ocupan los graduados de la carrera de Ingeniería de Sistemas, se identificó que el cargo con mayor porcentaje corresponde al área operativa, el $13,9 \%$ corresponde a jefaturas departamentales, mientras que sólo el 5,6\% corresponde a cargos gerenciales, lo que se justifica debido a que los Ingenieros de Sistemas tienen poco tiempo laborando en las organizaciones, ya que en el Ecuador la mayoría de las empresas colocan a empleados con más antigüedad y experiencia en cargos directivos.

Adicionalmente, en relación a la dificultad para conseguir empleo, en términos generales el mayor porcentaje del periodo indica que resulta fácil conseguir trabajo.

\section{CONCLUSIONES}

Al culminar el presente trabajo de investigación se puede considerar que de los graduados de la corte del año 2017 al 2019, el 85\% correspondiente a la muestra lo que resulta muy satisfactorio para esta evaluación, así se identificó que el profesional de la Carrera de Ingeniería de Sistemas se desempeña en actividades relacionadas a su perfil de egreso dentro del área operativa en el $80,6 \%$, esto debido a su reciente incorporación al mercado laboral. El sector que más oportunidades brinda al profesional en ingeniería en Sistemas de forma general es el sector privado pero también muchos egresados optan por emprender sus negocios, que tanto la Universidad como la Carrera han venido fomentado en estos últimos años. 
Mediante el estudio realizado se identificó que existen factores que afectan la empleabilidad de los estudiantes recientemente graduados, y que es necesario que la Universidad intervenga de alguna manera para incentivarlos a insertarse en el ámbito laboral. Además, hay que tener en cuenta la realidad sobre los empleos en el país, debido a que, para acceder a un puesto de trabajo el requisito básico es la experiencia en áreas similares, requisito que la mayoría de graduados no posee, por lo que se hace más dificultoso acceder de inmediato a un trabajo y en especial a un cargo de alto nivel.

En relación a la existencia de factores externos que intervienen en la empleabilidad, se identificó como causa principal la situación económica del país y al requisito de la experiencia requerido por la mayoría de empresas a nivel nacional. Además, existe una pequeña cantidad de graduados que no están trabajando de acuerdo a su perfil profesional, sin embargo, están desempeñando sus funciones a cambio de una remuneración básica, lo que constituiría un efecto de la situación del país que obliga a algunos graduados a estar en condiciones de subempleo.

Las Unidades de Vinculación en las Instituciones de Educación Superior, que realizan el seguimiento a graduados sobre la situación laboral, deben continuar apoyando a sus graduados y estimularlos a insertarse en el ámbito laboral con el objetivo de mejorar la calidad de vida de sus familias.

\section{RECOMENDACIONES}

Es indispensable que se puedan crear portales de empleo, dirigidos a los todos los estudiantes y graduados de las instituciones de Educación Superior en general, o a su vez el gobierno puede implementar programas que promuevan el emprendimiento e innovación en los jóvenes, aspectos que se han venido canalizando pero que no llega a todo nivel en especial a carreras técnicas como la de sistemas. En relación a los requisitos para otorgar empleos, se debería reestructurar a nivel normativo la participación en igualdad de oportunidades en el ámbito laboral tanto para hombres como para mujeres y personas con discapacidad estableciendo cuotas fijas que promuevan esa equidad $y$ verificando su cumplimiento, lo que a su vez permitiría motivar a más mujeres a estudiar carreras técnicas como Sistemas, que hoy en día se evidencia en este estudio, existen en número reducido mujeres que optan por cursar este tipo de estudios a nivel superior.

Las unidades de Vinculación de cada Institución de Educación Superior, no solo deberían realizar un seguimiento a los estudiantes, para sus prácticas o pasantías, a nivel de graduados se podría mantener activa la bolsa de trabajo que favorezca la vigilancia permanente y el contacto con empresas para poder insertar laboralmente de forma especial a sus graduados gracias a los convenios que se podrían establecer con empresas del sector informático, objeto especial de este caso de estudio. Debido a que generalmenteel estudiantegraduadosoloobtiene experiencia en las prácticas pre-profesionales o pasantías, una opción adicional sería que en la planificación curricular de la carrera se incrementen las horas de prácticas, para que de esa manera el estudiante pueda insertarse en el ámbito laboral con oportunidades que inicien desde los niveles intermedios de la Carrera y alcanzar así la tan requerida experiencia laboral. Otra alternativa que podría facilitar esta situación puede ser la inserción laboral temprana a través de las prácticas preprofesionales o pasantías exigidas por la Universidad, de forma tal que se permita a los estudiantes hacer más horas de prácticas o pasantías si así lo desean, para que así puedan adquirir la experiencia exigida por las empresas en el Ecuador cuando se gradúen y ésto se puede lograr sólo a través de una amplia bolsa de empresas con las cuales se 
hayan establecido convenios interinstitucionales para el efecto.

Debido a la situación general del país, lo principal sería inculcar a los estudiantes el espíritu emprendedor, debido a que existen diferentes oportunidades de negocio en el campo informático, las cuales pueden materializarse y a su vez convertirse en fuentes de empleo para otras personas.

\section{AGRADECIMIENTOS}

A la Carrera de ingeniería de Sistemas por otorgar el permiso para la realización de esta investigación. Al Departamento de Vinculación por permitirnos acceder a información relacionada al tema investigado.

\section{BIBLIOGRAFÍA}

Cristóbal, J. (2015). ALGUNAS REFLEXIONES ENTORNOAL PERFECCIONAMIENTO DEL DISEÑO CURRICULAR DE LA CARRERA DE INGENIERIA EN SISTEMAS. Revista Electrónica Formación y Calidad Educativa, 151160.

El Comercio. (25 de julio de 2019). Actualidad. Una guía de la Senescyt para elegir carrera universitaria en Ecuador circulará este domingo 28 de julio del 2019 con EL COMERCIO, pág. 1.

El Universo. (23 de septiembre de 2018). Noticias. $28 \%$ de los desempleados tienen estudios superiores, pág. 1.

El Universo. (17 de enero de 2020). Política. EI INEC dice que el empleo pleno en Ecuador cayó en el 2019, pág. 1.
Espinosa, F. D. (2018). Empleabilidad en el Ecuador desde una perspectiva de género. Consultora Económica, 23-33.

Gavilanes, J. E., \& Fernández, A. J. (2015). LA EMPLEABILIDAD DE GRADUADOS UNIVERSITARIOS EN EL CONTEXTO LATINOAMERICANO. REALIDADES $D E \quad$ UNIANDES, ECUADOR. Recuperado el 24 de 2 de 2020, de https://atenas.reduniv.edu.cu/index. php/atenas/article/view/169

Gonzáles, L. (01 de enero de 2020). Emagister. Obtenido de https://www.emagister. com/blog/que-hace-un-ingeniero-desistemas/

González, J. A. (2011). LA EMPLEABILIDAD: UNA COMPETENCIA PERSONAL Y UNA RESPONSABILIDAD SOCIAL. Recuperado el 24 de 2 de 2020, de https://ideas.repec.org/a/erv/coccss/ y2011i2011-123.html

Gonzalez, V. P. (2013). Propuesta para la evaluación de la empleabilidad inicial en ingeniería. Ciencia y Tecnología, 1(13), 403-416. Recuperado el 24 de 2 de 2020, de https://dialnet.unirioja.es/ servlet/articulo?codigo $=4843875$

Greciano, B. G. (2017). Empleabilidad: Un Enfoque Basado en las Competencias. Recuperado el 24 de 2 de 2020, de http://eprints.ucm.es/36044

INEC, I. N. (2016). Obtenido de https:// www. ecuadorencifras.gob. ec/documentos/web-inec/ EMPLEO/2016/Septiembre-2016/ Nota $\% 20$ metodologica $\% 20$ final $\% 20$ actualizada\%20(Septiembre-16).pdf 
Lantarón, B. S. (2016). Empleabilidad: análisis del concepto. Recuperado el 24 de 2 de 2020, de http://reined.webs4.uvigo.es/ index.php/reined/article/view/225

Martínez, A. A., \& Reyes, Y. M. (2018). EVALUACIÓN DE COMPETENCIAS DE PROGRAMACIÓN EN LA FORMACIÓN DEL INGENIERO EN SISTEMAS COMPUTACIONALES. Recuperado el 24 de 2 de 2020, de http://anfei.org.mx/revista/index.php/ revista/article/view/434

Novoa, F. A. (2016). INGENIERÍA DE SISTEMAS, LA CARRERA DEL PRESENTE. Recuperado el 24 de 2 de 2020, de http://revistas.ustatunja. edu.co/index.php/ingeniomagno/article/ view/1201

OEI. (2017). Organización de Estados Iberoamericanos. Obtenido de https:// www.oei.es/historico/oeivirt/fp/03a02. $\mathrm{htm}$

Pico, P. (2 de Febrero de 2015). Blog del Ingeniero de Sistemas. Obtenido de https://ingenierodesistemas.co/editorial/ definicion-de-ingenieria-de-sistemas/

Santacruz, D. (2007). El análisis ocupacional como estrategia de cambio en las organizaciones. Recuperado el 24 de 2 de 2020, de https://biblat.unam.mx/ es/revista/vision-gerencial/articulo/elanalisis-ocupacional-como-estrategiade-cambio-en-las-organizaciones

Senecyt. (3 de Marzo de 2020). Secretaria de Educación Superior, Ciencia, Tecnología e Innovación. Obtenido de https://www. educacionsuperior.gob.ec/mayoresoportunidades-para-el-acceso-a-la- educacion-superior-31-de-incrementoen-la-oferta-academica/

UniNorte. (2015). Universidad del Norte. Obtenido de https://www.uninorte. edu.co/web/ingenieria-de-sistemas-ycomputacion/perfiles

Universia. (20 de enero de 2020). Universia. Obtenido de Universia: https://noticias. universia.es/practicas-empleo/ noticia/2014/02/12/1081179/carrerascon-mas-futuro-2020.html

Valencia, A. (2004). La relación entre la ingeniería y la ciencia. Obtenido de Revista Facultad de Ingeniería Universidad de Antioquia, núm. 31: https://www.redalyc. org/pdf/430/43003113.pdf 